\title{
An update on the management of breast atypical ductal hyperplasia
}

\author{
Breast atypical ductal hyperplasia: new evidence and perspectives
}

\begin{abstract}
Among lesions with uncertain malignant potential found at percutaneous breast biopsy, atypical ductal hyperplasia $(\mathrm{ADH})$ carries both the highest risk of underestimation and the closest and most pathologist-dependent differential diagnosis with ductal carcinoma in situ (DCIS), matching the latter's features save for size only. ADH is therefore routinely surgically excised, but single-centre studies with limited sample size found low rates of upgrade to invasive cancer or DCIS. This suggests the possibility of surveillance over surgery in selected subgroups, considering the $2 \%$ threshold allowing for follow-up according to the Breast Imaging Reporting and Data System. A recent meta-analysis on 6458 lesions counters this approach, confirming that, surgically excised or managed with surveillance, ADH carries a $29 \%$ and $5 \%$ upgrade rate, respectively, invariably higher than $2 \%$ even in subgroups considering biopsy guidance and technique, needle calibre, apparent complete lesion removal. The high heterogeneity $\left(I^{2}=80 \%\right)$ found in this meta-analysis reaffirmed the need to synthesize evidence from systematic reviews to achieve generalizable results, fit for guidelines development. Limited tissue sampling at percutaneous biopsy intrinsically hampers the prediction of $\mathrm{ADH}$-associated malignancy. This prediction could be improved by using contrast-enhanced breast imaging and applying artificial intelligence on both pathology and imaging results, allowing for overtreatment reduction.
\end{abstract}




\section{Atypical ductal hyperplasia and its management}

In clinical practice, the use of core needle biopsy (CNB) and vacuum assisted biopsy (VAB) before surgery has long replaced surgical breast biopsy, which is still used for diagnostic purposes in few cases only. This transition engendered both advantages (reduced costs, postbiopsy complications and mammographic changes) and disadvantages. ${ }^{1}$ The latter stem from the forcibly incomplete lesion sampling, ${ }^{2}$ which hinders histopathologic interpretation and increases the risk of underestimating malignancy ${ }^{3}$ - especially with small-calibre CNB needles $^{1-3}$ - and of having an indeterminate (B3) lesion diagnosed at pathology. ${ }^{2,4}$ Heterogeneity in the wide group of B3 lesions, which includes atypical ductal hyperplasia (ADH), flat epithelial atypia, atypical lobular neoplasia, lobular carcinoma in situ, benign phyllodes tumour, radial scars and papillary lesions, is mirrored by high variations in their still undefined malignant potential. ${ }^{2,3}$

$\mathrm{ADH}$ is one of the most frequent B3 lesions (along with papillary lesions and flat epithelial atypia) and the one associated to the highest increase in breast cancer risk. ${ }^{3,5}$ Debate on B3 lesions management is centered on the concept of upgrade rate, i.e. the rate of percutaneously-diagnosed lesions which, after surgical excision or during active imaging surveillance, result into ductal carcinoma in situ (DCIS) or invasive cancers. ${ }^{2,3,5}$

In the past, all percutaneously-diagnosed B3 lesions underwent surgical excision to avoid underestimation. ${ }^{2,6}$ Since this approach led to overtreatment in a high rate of cases, ${ }^{5-7}$ a new approach based on surveillance has been advocated for various B3 lesions subtypes. ${ }^{2,6-8}$ For example, surveillance could be carried out with whole-breast morphofunctional imaging techniques such as contrast-enhanced MRI (CE-MRI) ${ }^{9}$ or contrast enhanced mammography $(\mathrm{CEM}),{ }^{10}$ which have recently shown promising results, albeit in single-centre retrospective studies on a limited number of patients. 
However, the benefit-cost ratio of this surveillance-based approach in women with ADH remains even more unclear than for other B3 lesions. To adopt the surveillance option, we should consider a $2 \%$ upgrade rate threshold to justify the avoidance of surgical excision, ${ }^{11}$ in parallel to the Breast Imaging Reporting and Data System (ACR BI-RADS) category 3. ${ }^{12}$ However, this threshold remains debatable, as showed by the results of the Second International Consensus Conference on lesions of uncertain malignant potential in the breast (B3 lesions), ${ }^{6}$ in which participants were asked what they thought to be an acceptable underestimation rate for DCIS or invasive cancer. Almost half of the participants indicated a $5 \%$ threshold for invasive cancer and $78 \%$ of them a $10 \%$ threshold for DCIS. Such a difference remains highly relevant and warrants discussion.

The aim of this paper is to summarize evidence on ADH management, comparing opposite conclusions of a single-centre retrospective study on VAB versus surgical biopsy published in this journal in $2018^{13}$ and of a recently published meta-analysis on 6458 ADHs. ${ }^{11}$

\section{Small trials versus larger evidence}

Already in 2002, Halpern et al ${ }^{14}$ cited various reports in which small and statistically underpowered randomized trials were deemed "scientifically useless" and "unethical", warning to cautiously consider them and extending these concerns to single-centre observational retrospective studies. In the setting of critical care, Bellomo et al ${ }^{15}$ further compared single-centre trials to a Trojan horse, advising against their use in guidelines development.

However, Halpern et al $^{14}$ also highlighted how conclusions from small studies could still be useful (and ethically justified) for rare diseases, since poorly-generalizable results can be combined in meta-analyses. Studies focusing on B3 lesions are intrinsically prone to the aforementioned shortcomings. Their unknown malignant potential ${ }^{2,3}$ and low concordance 
between pathologists in their assessment further complicates this scenario, ${ }^{4}$ especially for ADH that has histopathologic features akin to low-grade DCIS., ${ }^{2,5}$

\section{Vacuum assisted breast biopsy (VAB) excision of microcalcifications with extension lower than one centimetre as an alternative to open biopsy for atypical ductal hyperplasia}

In 2018, our group published a study on 65 percutaneously-diagnosed ADH on a single group of microcalcifications - completely removed after $\mathrm{VAB}^{13}$ - considering results of a 7-year series with a mean follow-up higher than 5 years. Its conclusions favoured surveillance with mammography without surgical excision in a highly selected group of patients, i.e. patients with a percutaneously-diagnosed ADH, on a single group of BI-RADS $4 \mathrm{~b}$ microcalcifications sampled with VAB under stereotactic guidance, without apparent post-procedure residual and without multiple hyperplastic foci or high percentages of hyperplasia at histopathological assessment. These results substantially agreed with the First International Consensus Conference, in which $42 \%$ of all voting participants ( 27 radiologists, 2 pathologists, 2 surgeons, 16 gynaecologists) thought that surveillance was adequate for ADHs diagnosed by $\mathrm{VAB}^{8}$

\section{The First and Second International Consensus Conferences: a changed perspective?}

However, recommendations from the Second International Consensus Conference markedly differed from the first ones. ${ }^{6}$ While the first recommendation on ADH was "A lesion containing ADH which is visible on imaging should undergo therapeutic open surgical excision. If a unifocal ADH lesion has been completely removed by $V A B$, surveillance is

justified. Otherwise open surgery is still recommended", 8 the second one was: "A lesion containing ADH diagnosed by CNB or VAB should undergo open surgical excision. 
Surveillance can be justified only in special situations after discussion at the multidisciplinary meeting". 6

\section{New evidence}

These contrasting recommendations, initially appearing as a step-back towards more aggressive treatment, prompted us to follow Halpern et al ${ }^{14}$ suggestions synthesizing available literature on $\mathrm{ADH}$, rich in small single-centre studies. We performed a meta-analysis on 93 studies with a total of 6458 ADH lesions, aiming to identify the group of patients in whom surgery could be avoided. ${ }^{11}$ This was however not the case: even considering only the upgrade rate to invasive ductal carcinoma (IDC) after surgical excision or during follow-up without surgical excision, data from the included studies did exceed the aforementioned $2 \%$ threshold, to justify surveillance-based management. We obtained a pooled upgrade rate of $29 \%$ (95\% confidence interval [CI]: 26\%-32\%) for surgically-excised lesions and 5\% (95\% CI: $4 \%-8 \%$ ) for those managed with follow-up. ${ }^{11}$ Moreover, we found an upgrade rate of 14\% (95\% CI: $8 \%-23 \%$ ) for completely removed lesions. ${ }^{11}$ This figure is completely at odds with recommendations from the First International Consensus Conference, ${ }^{8}$ while however agreeing with the Second International Consensus Conference, that excluded or - better reduced the potential role of surveillance without surgical excision for percutaneouslydiagnosed $\mathrm{ADH}^{6}$

Moreover, as pointed out by the Second International Consensus Conference, ${ }^{6}$ the use of different thresholds for invasive cancer and DCIS is advisable. As already mentioned, we therefore performed subgroup analyses independently examining upgrade rates to IDC and DCIS. ${ }^{11}$ For IDC, we obtained an upgrade rate of $9 \%$ (95\% CI: $7 \%-11 \%$ ) for surgicallyexcised lesions and 3.4\% (95\% CI: 1.8\%-6.4\%) for those managed with follow-up. For DCIS, 
we found an upgrade rate of 20\% (95\% CI: 18\%-23\%) for surgically-excised lesions and $2.8 \%$ (95\% CI: $1.5 \%-5.1 \%$ ) for those managed with follow-up.

Finally, as already postulated in our retrospective paper, ${ }^{13}$ our meta-analysis showed how an upgrade rate higher than $2 \%$ was independent of biopsy technique (CNB or VAB), needle calibre, imaging guidance (stereotactic, ultrasound or magnetic resonance imaging) and of apparent complete lesion removal. ${ }^{11}$

\section{Conclusions}

The opposite results from our papers are paradigmatic of different evidence levels: unfiltered information from small single-centre studies constitute the base of the evidence-based medicine pyramid, while the apex is represented by filtered information from meta-analyses. While with the first paper we intended to publish findings from our centre, recognizing their low generalizability, the second study aimed to find selective conditions that could be generalizable and guide ADH management reducing overtreatment. Unfortunately, we did not find any parameter that, at present, could stratify patients and justify a surveillance-based management. ${ }^{5,11}$ This is not what the scientific community is looking for, since we are still accepting a certain level of unwanted overtreatment that could be reduced or even eliminated by pursuing two different strategies.

The first strategy is based on second opinion pathology interpretation, as already performed in some centres and proposed by various authors, such as Tosteson et al, ${ }^{16}$ who found lower overtreatment for atypia cases (1.8\%) with a second opinion versus cases without (4.7\%), emphasizing how this approach also reduces undertreatment and overall care costs

The second strategy envisages the use of artificial intelligence, as postulated by Bahl et al, ${ }^{17}$ who applied machine learning to predict the pathological upgrade of B3 lesions to DCIS and invasive cancers, aiming to reduce unnecessary surgical excision. The authors used 
different parameters to classify low-risk and high-risk lesions, including patient age, B3 characteristics and full-text analysis of the pathologic report, considering a resulting $5 \%$ or higher probability of upgrade as a threshold warranting surgical excision. The application of this approach on the internal test dataset of 335 B3 lesions would have significant reduced unnecessary surgeries by $25 \%$ compared to the authors' institutional practice and correctly identified $97 \%$ of all B3 lesions with a subsequent upgrade. Even though needing external validation, this is a promising approach.

In conclusion, current approaches towards a better management of B3 lesions, in particular $\mathrm{ADH}$, are intrinsically hindered by limited tissue sampling, given the potential distance between B3 lesions and associated DCIS or invasive cancer. ${ }^{2}$ Future research integrating CEM or CE-MRI with artificial intelligence models could lead to precision decision-making, reducing overtreatment. 


\section{References}

1. van Breest Smallenburg V, Nederend J, Voogd AC, Coebergh JWW, van Beek M, Jansen FH, et al. Trends in breast biopsies for abnormalities detected at screening mammography: a population-based study in the Netherlands. Br. J. Cancer 2013; 109: 242-48. doi: 10.1038/bjc. 2013.253

2. Sharma N, Wilkinson LS, Pinder SE. The B3 conundrum - the radiologists' perspective. Br. J. Radiol. 2017; 90: 20160595. doi: 10.1259/bjr.20160595

3. Forester ND, Lowes S, Mitchell E, Twiddy M. High risk (B3) breast lesions: What is the incidence of malignancy for individual lesion subtypes? A systematic review and metaanalysis. Eur. J. Surg. Oncol. 2019; 45: 519-27. doi: 10.1016/j.ejso.2018.12.008

4. Elmore JG, Nelson HD, Pepe MS, Longton GM, Tosteson ANA, Geller B, et al. Variability in Pathologists' Interpretations of Individual Breast Biopsy Slides: A Population Perspective. Ann. Intern. Med. 2016; 164: 649. doi: 10.7326/M15-0964

5. Brem RF. Management of Breast Atypical Ductal Hyperplasia: Now and the Future. Radiology 2020; 294: 87-88. doi: 10.1148/radiol.2019192192

6. Rageth CJ, O’Flynn EAM, Pinker K, Kubik-Huch RA, Mundinger A, Decker T, et al. Second International Consensus Conference on lesions of uncertain malignant potential in the breast (B3 lesions). Breast Cancer Res. Treat. 2019; 174: 279-96. doi: 10.1007/s10549-01805071-1

7. Sardanelli F, Trimboli RM, Tot T. Expert Review of Breast Pathology in Borderline Lesions. JAMA Oncol. 2018; 4: 1325. doi: 10.1001/jamaoncol.2018.1953

8. Rageth CJ, O’Flynn EA, Comstock C, Kurtz C, Kubik R, Madjar H, et al. First International Consensus Conference on lesions of uncertain malignant potential in the breast (B3 lesions). Breast Cancer Res. Treat. 2016; 159: 203-13. doi: 10.1007/s10549-016-3935-4 9. Bertani V, Urbani M, La Grassa M, Balestreri L, Berger N, Frauenfelder T, et al. Atypical 
ductal hyperplasia: breast DCE-MRI can be used to reduce unnecessary open surgical excision. Eur. Radiol. 2020. doi: 10.1007/s00330-020-06701-3

10. Bicchierai G, Nori J, De Benedetto D, Boeri C, Vanzi E, Bianchi S, et al. Follow- up of B3 breast lesions without residual microcalcifications post vacuum- assisted biopsy, can contrast- enhanced digital mammography help? Breast J. 2020; 26: 299-302. doi: $10.1111 /$ tbj. 13598

11. Schiaffino S, Calabrese M, Melani EF, Trimboli RM, Cozzi A, Carbonaro LA, et al. Upgrade Rate of Percutaneously Diagnosed Pure Atypical Ductal Hyperplasia: Systematic Review and Meta-Analysis of 6458 Lesions. Radiology 2020; 294: 76-86. doi: 10.1148/radiol.2019190748

12. D’Orsi CJ, Sickles EA, Mendelson EB, Morris EA. ACR BI-RADS® Atlas, Breast Imaging Reporting and Data System. Fifth. Reston, VA: American College of Radiology; 2013.

13. Schiaffino S, Massone E, Gristina L, Fregatti P, Rescinito G, Villa A, et al. Vacuum assisted breast biopsy (VAB) excision of subcentimeter microcalcifications as an alternative to open biopsy for atypical ductal hyperplasia. Br. J. Radiol. 2018; 91: 20180003. doi: 10.1259/bjr.20180003

14. Halpern SD, Karlawish JHT, Berlin JA. The Continuing Unethical Conduct of Underpowered Clinical Trials. JAMA 2002; 288: 358. doi: 10.1001/jama.288.3.358 15. Bellomo R, Warrillow SJ, Reade MC. Why we should be wary of single-center trials. Crit. Care Med. 2009; 37: 3114-19. doi: 10.1097/CCM.0b013e3181bc7bd5 16. Tosteson ANA, Yang Q, Nelson HD, Longton G, Soneji SS, Pepe M, et al. Second opinion strategies in breast pathology: a decision analysis addressing over-treatment, undertreatment, and care costs. Breast Cancer Res. Treat. 2018; 167: 195-203. doi:

$10.1007 / \mathrm{s} 10549-017-4432-0$ 
17. Bahl M, Barzilay R, Yedidia AB, Locascio NJ, Yu L, Lehman CD. High-Risk Breast

Lesions: A Machine Learning Model to Predict Pathologic Upgrade and Reduce Unnecessary

Surgical Excision. Radiology 2018; 286: 810-18. doi: 10.1148/radiol.2017170549 